\title{
Editorial
}

Songfa Zhang ${ }^{1}$, Shan Jiang ${ }^{2 \star}$, Xiao Zang ${ }^{3}$

\section{A promising measure to prevent HPV-positive oral squamous cell carcinoma}

DOI: 10.1515/ii-2017-0156

Received September 6, 2017; accepted September 12, 2017; published online November 28, 2017

The article "HPV and oral squamous cell carcinoma: a review of HPV-positive OSCC and possible strategies for future" provides inspiring insights into the association between human papillomavirus (HPV) infection and oral squamous cell carcinoma (OSCC), as well as the possible strategies that we can adopt in future to minimize its health impact on the population [1]. This association has been widely recognized in recent years, particularly, for the high-risk HPV 16 and 18 strains. The cancer-inducing proteins E6 and E7 of HPV have been identified to be the main factors that inhibit the tumor suppressors $\mathrm{p} 53$ and retinoblastoma protein $(\mathrm{pRb})$, contributing to the development of OSCC. It also has been confirmed that HPV infection is associated with OSCC prognosis. Compared to HPV-negative OSCC patients, HPV-positive OSCC patients have a better prognosis [2].

At present, there is no suitable OSCC HPV infection detection method approved by the health administration. Potential methods include immunohistochemical detection of p16 expression, polymerase chain reaction (PCR), or in situ hybridization detection method, which are used to indirectly reflect the HPV infection status. Due to the ineffectiveness of these methods, there are no HPV infection status screening programs for the OSCC high-risk group. Therefore, developing effective HPV detection methods for the early detection and treatment of HPV-induced OSCC is of great significance.

HPV vaccine has been listed by the US Food and Drug Administration (FDA) for $>10$ years. We have witnessed the development of the HPV vaccine from the initial stage of protection against two HPV strains (HPV 16 and 18) to much wider protection against nine strains (HPV 6, 11, 16, 18, 31, 33, 45, 52, and 58). The coverage of pathogenic HPV has increased from $70 \%$ to $90 \%$. HPV vaccines play an important role in the prevention of HPV infection in the reproductive tract. However, the role of HPV vaccine in the prevention of oral HPV infection remains to be further investigated. It is still unclear whether the vaccine has effects on oral HPV infection [1]. There is also a lack of research on this topic. Studies with large sample sizes and on both genders are needed for the confirmation of the protective effects.

In the recent 10 years, the incidence of OSCC has increased. This fact has reinforced the necessity for research into clarification of the relationship among oral HPV infection, HPV-positive OSCC, and HPV vaccines. This article makes this question clearer and discusses possible strategies, considering that the association between HPV vaccine and HPV-positive OSCC is confirmed [1].

The most effective protective measure for HPV infection is the HPV vaccine [3]. However, people's participation in HPV vaccination programs is significantly lower than for other vaccines. Based on statistics from the US, in 2015, the HPV vaccination participation rate was $41.9 \%$ and $28.1 \%$, respectively, among girls and boys aged 13-17 years. These rates are much lower than the target set by the US, which expects an 80\% participation rate among the population aged 13-17 years in 2020. One reason is the lack of awareness of HPV-related diseases, such as HPV-positive OSCC, among the population. In addition, parents are concerned that their children will become more likely to have casual sex frequently with different partners when they are protected by HPV vaccine.

\footnotetext{
${ }^{1}$ Department of Gynecologic Oncology, Zhejiang Women's Hospital, School of Medicine, Zhejiang University, Hangzhou, China ${ }^{2}$ School of Population and Public Health, University of British Columbia, Vancouver, British Columbia V6T1Z3, Canada

${ }^{3}$ Faculty of Medicine, Simon Fraser University, Vancouver, British Columbia, Canada

*Correspondence: Shan Jiang

E-mail: shan.jiang@ubc.ca
} 
To achieve the $80 \%$ aim, governments and scientists should work together to increase the awareness of HPV-related diseases. It is crucial to make the population aware that every person is likely to be infected by HPV in his/her lifetime, and that HPV infection is associated with many diseases. Other measures include providing coverage in health insurances and making the HPV vaccination a mandated program for children.

Authors' Contributions: Zhang wrote the first version of this article. Jiang revised it and wrote the second version. Zang provided critical contents for the final version. The three authors had sufficient discussion before they arrived at the final version.

Acknowledgments: We thank our colleagues from Zhejiang Women's Hospital, University of British Columbia, and Simon Fraser University. The daily discussions with them inspired us and helped to form a series of articles on HPV and HPV vaccines.

Conflict of Interest: Authors state no conflict of interest.

\section{References}

[1] Jiang S., Dong Y., HPV and oral squamous cell carcinoma: a review of HPV-positive OSCC and possible strategies for future, Current Problems in Cancer, 2017, 5,323-327.

[2] Forastiere A.A., Ang K., Brizel D., Brockstein B.E., Dunphy F., Eisele D.W., et al., Head and neck cancers: clinical practice guidelines, J. Natl. Compr. Cancer Network, 2005, 3, 316-391.

[3] Daley E.M., Vamos C.A., Thompson E.L., Zimet G.D., Rosberger Z., Merrell L., et al., The feminization of HPV: how science, politics, economics and gender norms shaped US HPV vaccine implementation, Papillomavirus Res., 2017, 3, $142-148$. 